Dept. of Forensic Medicine \& Toxicology

Fac. Vet. Med., Assiut University

Head of Dept.: Prof.Dr.Abd El-Aziz Shaaban.

\title{
HEMATOLOGICAL AND BIOCHEMICAL STUDIES OF FLUORINE POISONING IN CHICKENS, A TRIAL FOR TREATMENT (With 10 Tables)
}

\author{
By \\ A. SH. SEDDEK,TH. A. IBRAHIM, \\ $A H L A M A B D E L-H A M I D^{*}$ and M. ABD EL-NASSER
}

* Animal Health Research Institute, Dokki, Giza.

(Received at 17/12/1996)

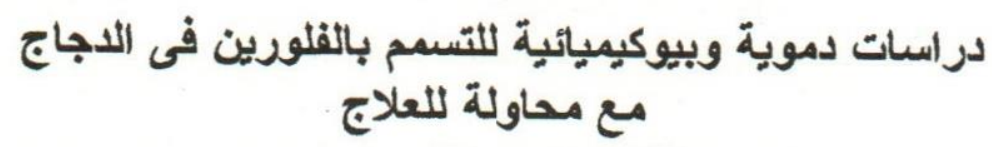

عبل اللطبف شاكر ، ثابت عبل المنعم ، أحلام عبل الحمبل ، محصود عبل الناصر

تم أجر اء هذا البحث لدراسه الاثار السميه لفلوريد الصوديوم على الاجساج ومّد تم أستعمال مانسة

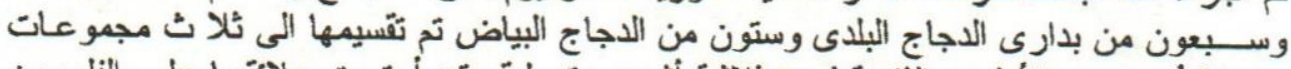

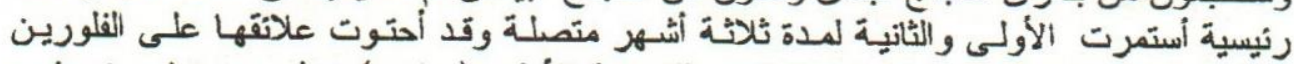

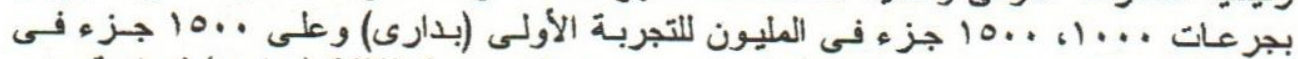

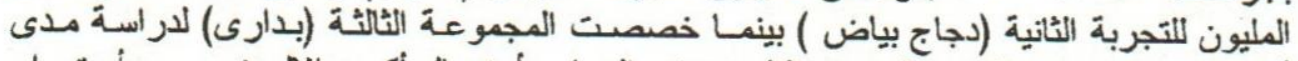

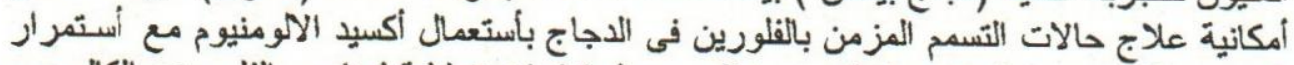

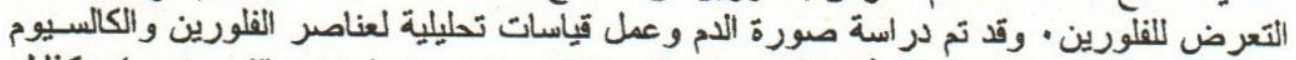

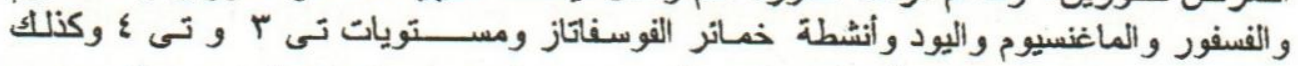

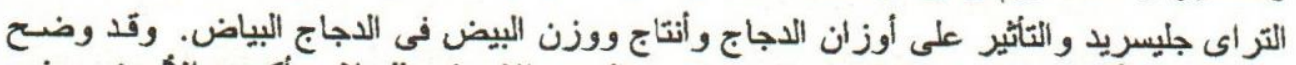

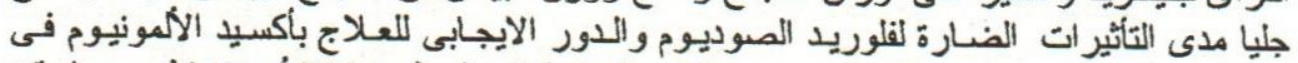

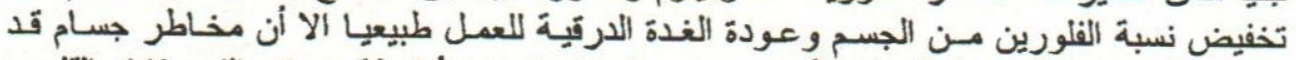

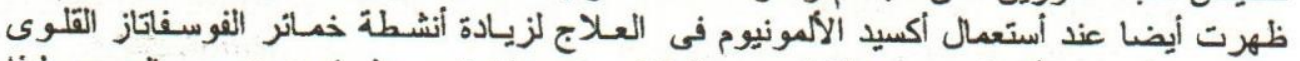

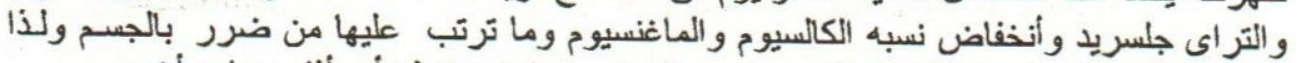
فلابد من أستمر ار المحاو لات ألعلاجيه حتى يمكن تعميمها دون ترك ألى أثلى أثرار ضاره أخري. 


\section{SUMMARY}

This study was carried out to investigate the toxic and hazardous effects of sodium fluoride $(\mathrm{NaF})$ on chickens. One hundred and seventy Balady broiler and sixty laying hens have been used in this study. Three experiments were conducted to achieve this goal. In the first experiment, two groups of balady chickens were fed on two doses (1000 and 1500 ppm) of $\mathrm{NaF}$ and third group served as control for three months. In the second experiment, two groups of white high line laying hens were used The first group was treated with $1500 \mathrm{ppm} \mathrm{NaF}$ while the second was kept for three months as control. In the third experiment, balady broiler chicken previously intoxicated by sodium fluoride $(1000,1500 \mathrm{ppm})$ for three months were used and treated with alluminum oxide. Hematological picture and biochemical analysis of Fluorine, Calcium, Phosphorous, Magnesium, Iodine, Triiodothyronine (T3), Thyroxine (T4), Alkaline Phosphates activity, and Triglycride revealed highly significant variation than normal. Also, mean egg weight, feed consumption, and body weight gain were decreased. The use of aluminum oxide has some beneficial effects for treatment of florine toxicity in broiler chicken.

Key words: Fluorine poisoning - Chickens - Treatment.

\section{INTRODUCTION}

Fluorides are salts of hydrogen fluoride. The element fluorine is the most electronegative of all elements. Its affinity to a number of elements is very considerable. Due to the increase uses of fluorides in the treatment of certain metabolic bone diseases, particularly Osteoporosis in one hand and its application as feed additives as calcium source and as a result of ecosystem contamination from industrial pollution specially by super-phosphate factory on another hand, the present experiments were conducted to determine the exerted toxic effect of fluorine on laying hens with special interest to their performance.Abdel Nasser et al (1995) reported a highly significant reduction in body weight and body weight gain in fluorine treated chickens They concluded in their work that $\mathrm{NaF}$ alter the immune response and reduce host resistance to infection. Hasanien et al. (1995) recorded chromosomal aberrations in chickens treated with $\mathrm{NaF}$. As fluorine has tendency to combine with other elements, the experiment included the picture of most essential elements that related strongly to fluorine in addition to the thyroid hormones and iodine. The application of aluminum compounds as an 
alleviating elements for fluorosis had a special interest. So an experiment was done to detect the efficiency of aluminum (AI) on the exerted toxic effects of fluorine toxicosis either on the hematological picture, thyroid hormones, or on related elements as $\mathrm{Ca}, \mathrm{P}, \mathrm{Mg}$ and Iodine. The aim of this study was to put a clear line on the application of fluoride in one hand and the alleviation of its effect by using Aluminum. oxide.

\section{MATERIALS and METHODS}

One handred and seventy Balady broilers and sixty laying hens have been used in this study in three experiments.

\section{Experiment I:}

In this experiment, 90 clinically healthy Balady broilers were used, vaccinated against Newcastle, free from internal or external parasites, weighing 400-550 g, and aging two months. These chickens were classified into three groups, the first two groups fed a ration containing 1000 and 1500 ppm of sodium fluoride for 3 months and the third group was kept as control, each group contained 6 males and 24 females.

\section{Experiment II:}

Sixty apparently healthy, white high line laying hens of six months, age weighing from 1.00 to $1.500 \mathrm{~kg}$ were used. Birds were classified into two groups, each of them contained 20 bird ( 4 males and 16 females). The first group fed a ration containing $1500 \mathrm{ppm}$ of $\mathrm{NaF}$ for three months while the second was used as a control.

\section{Experiment III:}

Eighty Balady broilers of five months age were used in this experiment. These chickens were classified into five groups, the first two groups fed a ration containing 1000 and $1500 \mathrm{ppm}$ of sodium fluoride for 4 weeks, the third and fourth groups fed a ration containing $1000 \mathrm{ppm}$ sodium fluoride + $8000 \mathrm{ppm}$ aluminum oxide and $1500 \mathrm{ppm}$ sodium fluoride $+12000 \mathrm{ppm}$ aluminum oxide for 4 weeks while the fifth group was kept as control, each group contained 4 males and 16 females.

The three experiments were performed in an environmentally controlled daylighted laboratory. Tape water $(0.02 \mathrm{ppm}$ fluoride $)$ was provided ad libitum. Commercial ration was used in the feeding of birds containing $3.60 \pm 0.26 \mathrm{ppm}$ fluoride.

\section{Chemicals:}

Sodium fluoride ( $99 \%$ purity), Aluminum oxide ( $99 \%$ purity) were purchased from Aldrich chemical company Ltd. 


\section{Assiut Vet. Med. J. Vol. 36 No. 72 , January 1997}

\section{Sampling:}

Blood samples were obtained from wing veins for hematological examination and serum samples were taken 1,2 and 3 months after exposure of broilers to fluoride in the $1^{\mathrm{st}}$ experiment for estimation of serum contents of fluoride, calcium, phosphorous, magnesium, triglycerides, iodine, triiodothyroxine, thyroxin, and alkaline phosphatase activity; 1, 2 and 3 months for laying hens previously exposed to $1500 \mathrm{ppm}$ of fluoride in experiment II and 1, 2, 3 and 4 weeks in the third expirment were administered to the same parameters.

\section{Methods:}

Red blood cells (RBCs), White blood cells (WBCs), Hemoglobin concentration (HB), packed cell volume (PCV), Mean corpuscular volume (MCV), Mean corpuscular hemoglobin $(\mathrm{MCH})$, and Mean corpuscular hemoglobin concentration (MCHC) were determined according to methods cited by Coles (1986),

Fluorine, calcium, inorganic phosphorous, magnesium and iodine serum levels were determined according to Fry and Taves (1970), Bett and Fraser (1959), Mornil and Prox (1973), Grinder and heth (1971) and Morin et al. (1975) respectively.

Serum triglyceride concentration, and alkaline phosphatase activity were determined according to the methods optained by Fletcher (1968) and Balfiedld and Goldberge (1971) .Quantitative determination of T3, and T4 in serum were obtained after Zoasoo (1975). Statistical analysis of data was performed after Kalton (1967).

\section{RESULTS and DISCUSSION}

Fluorine element (Tables 6, 7, 8) was increased to reach the toxicologic level which (over $0.5 \mathrm{ppm}$ ) indicates recent fluorosis (Greenwood et al., 1964) at the beginning of the second month of the second experiment (Table 8$)$. Fluorine level $(0.988 \mathrm{ppm})$ increased to reach the hazardous level at the $1^{\underline{\mathrm{st}}}$ week in the $1^{\mathrm{st}}$ experiment (Table 7) at $1500 \mathrm{ppm}$ dose level, meanwhile in the group treated with $1000 \mathrm{ppm}$ fluorine, the level were increased above toxic level at the end of the $4^{\text {th }}$ week $(0.53 \mathrm{ppm})$. Elevated levels of fluorine had been detected in hens exposed to industrial fluorosis at Manqubad area (Ahlam et al., 1994).

The effect of aluminum oxide as a line of treatment of fluorosis in chicken was observed in the form of decrease in fluorine levels during the 4 weeks in aluminum treated group than the levels in fluorosed control hens. This fact of decreased plasma fluorine had been described by Spencer et al. 
(1980). This decrease in plasma fluorine level could be attributed to the inhibition of intestinal fluorine absorption (Spencer and Lender, 1979). Aluminum compounds are the most frequently used alleviators of fluorosis (Becker et al., 1950). The effect of $\mathrm{Al}$ on Phosphorus appeared to inhibit its absorption from intestine. Phosphorus levels in treated group exposed to $1000 \mathrm{ppm}$ was decreased while birds exposed to $1500 \mathrm{ppm}$ showed increased levels in comparison to non treated fluorosed one. It was postulated that $\mathrm{Al}$ combines with phosphates in the intestine and induce phosphorus depletion which associated with increased bone resorption (Spencer and Lender, 1979; Brudevold et al., 1972 and Lotz et al., 1968).

Iodine levels increased in all hens exposed to fluorine either in the level of 1000 or $1500 \mathrm{ppm}$ whatever, young or adult, treated or non treated. This increase in iodine levels is cotradictionary to the finding of Ahlam et $\underline{\text { al., }}$, (1994) in endemic fluorosed hens present in the area of superphosphate producing plant. Thyroxin and Triiodothyronine (T4 \& T3) elevated levels indicates a stimulant effect of fluorine on the thyroid gland. This increase in both hormones in addition to the increased level of iodine in serum needs more work for the clarification of this stimulating effect of fluorine.

Triglycerides indicated a highly significant increase in the serum of exposed hens whatever treated or not. The effect of fluorine on triglycerides was detected in Guinea pigs after inhalation of HF where the triglycrides synthesis was accelerated in the liver and the extrahepatic lipoprotein lipase activity was also decreased in the fluoride group; this could be related to the decrease in apo protein C (Douset et al., 1984). The previous findings could explain the plasma triglycerides accumulation observed after exposure to $\mathrm{NaF}$ in all exposed hens.

The analysis of magnesium in serum of fluorine exposed hens revealed an increase in all treated and non treated groups. This result is in accordance with Seddek (1988) who found an increase in $\mathrm{Mg}$ in serum of goats showing endemic fluorosis. This increase was positively correlated to the increase in bone $\mathrm{Ca}$ and $\mathrm{Mg}$.

The analysis of $\mathrm{Ca}$ in blood serum revealed an increase in $\mathrm{Ca}$ levels in the $1^{\underline{\text { st }}}$ week in the 1000 and $1500 \mathrm{ppm}$ level while the use of aluminum led to a significant decrease in $\mathrm{Ca}$ levels. Calcium levels were decreased after one month of exposure. The decrease of $\mathrm{Ca}$ level in aluminum treated groups were evident during the $4^{\text {th }}$ weeks of exposure. Although $\mathrm{Ca}$ is particularly ameliorative, and no other cation can substitute for required $\mathrm{Ca}$, it seems unlikely that aluminum involves simple displacement of $\mathrm{Ca}$ from $\mathrm{Ca}$-requiring binding sites (Deleers, 1985). In the other hand $\mathrm{Ca}$ increased during $1^{\text {st }}$ 
month of adult exposed, the values decreased in the $2 \frac{\text { nd }}{}$ and $3^{\text {rd }}$ month of exposure. This could be related to the inhibition of $\mathrm{Ca}$ absorbance from the intestine due to $\mathrm{CaF}$ combination. Hatfield et al. (1942) found a considerable decrease in blood $\mathrm{Ca}$ with increased fluorine intake.

Alkaline phosphatase showed increased activity in the birds of the three experiments where the increase appeared in the $1^{\text {st }}$ week, this finding ensure that fluorine act on periostium which is the main source of AP enzyme, and the hyperactivity of the periostium is considered the main cause of increased AP activity (Rosenberger et al., 1979). This finding is in agreement with that of increased AP in experimentally exposed cows to fluorosis by Potmann (1979) who found an increase in AP activity in cows exposed to fluorine in rations over one year exposure to $\mathrm{CaF}$.

The hematological picture of hens in the $1^{\underline{s t}}$ experiment showed a highly significant decrease in RBCs number. The decrease was evident along the 3 months period. The dose $1500 \mathrm{ppm}$ showed more effect on inducing anemia. PCV values were non significantly decreased in the $1^{\mathrm{st}}$ month while the values were unchanged in the $2^{\frac{\text { nd }}{-}}$ and $3^{\text {rd }}$ month. Hemoglobin values were decreased which inversely correlated to the exposure dose. The mean corpuscular volume show a highly significant increase in all exposed birds indicating that anemia in this type of exposure of a macrocytic hypochromic one. The results of mean corpuscular hemoglobin concentration ensure this finding of hypochromacia which appeared clearly in the higher doses. In the second experiment the changes behave the same way in RBCs number, PCV, and hemoglobin concentration. In the $3 \frac{\text { rd }}{\text { experiment, the changes in total }}$ number of RBCs appeared in the 2 nd week, hemoglobin showed no significant change, but MCHC values decreased at $3 \frac{\text { rd }}{}, 4^{\text {th }}$ week. The PCV values showed a decrease correlated with the dose allover the test period. The AL treatment appeared to have no therapeutic effect on blood constituents in this part of experiment. The white blood cells showed no significant changes allover the exposure time in all the three experiment. It is apparent that there is no effect of fluorine on the WBCs. The change in RBCs had been recorded in cattle in India where the most significant results of fluorosis was the continuous fall in the hemoglobin and red cell contents of blood (Majumdar and Ray, 1946) Slagvold (1934) in sheep. Shupe et al. (1963) showed that fluorine had no effect on blood or haemobiotic centers. Potmann (1979) found no significant changes in blood constituents in cows fed $\mathrm{CaF}$ in ration for one year. The exerted effect in our result could be referred to the high dose used in ration(1000, $1500 \mathrm{ppm})$. 
The effect of fluorides on body weight and productive activity have been shown before (Schmidt et al. 1954) who found no significant differences in the milk or butter fat production of the controls or the animals fed various amounts of sodium fluoride. In the same time fluorine exerted no detrimental effect upon weight during the $1^{\text {st }}$ thirty six months of experiment. No differences could be detected in milk cow's yield and productivity with respect to weight gain and lactation in cows fed one year $\mathrm{CaF}$ in ration (Potmann, 1979). Our results showed a significant effect of fluoride as $\mathrm{NaF}$ in ration on body weight gain at a dose of $1500 \mathrm{ppm}$ from the $1^{\text {st }}$ week and in the $2 \frac{\text { nd }}{}$ week for 1000 ppm ration. This result could be attributed to less feed consumption which also decreased. Egg production and weight were decreased in fluorosed birds than control. In the same time application of aluminum as an alleviating element has a significant effect on correction of Fluoride, Iodine, T3, and T4 levels beside egg production in fluorosed hens. On other hand application of aluminum oxide in the treatment has undesirable effect on the calcium, and triglysride levels as well as on alkaline phosphatase activity. Body weight gain (Table 10) appeared to be not affected with aluminum addition to the diet during the 4 weeks of experiment.

In conclusion the previous findings explain the toxic effects of exposure to 1000 and $1500 \mathrm{ppm}$ fluorine. Aluminum as an alleviating ion to fluoride had diminished its level in serum but had no effect on its exerted toxic effect of decreasing $\mathrm{Ca}, \mathrm{Mg}, \mathrm{p}$ in serum. Aluminum had also no effect on the treatment of the hematological changes. The changes in egg weight, egg production, body weight gain were also not significantly affected by the addition of $\mathrm{AI}$ to ration. The ill response of the aforementioned exerted toxic effects of fluorides to aluminum compound could clarify that the use of aluminum must be in rations containing $1000 \mathrm{ppm}$ as a maximum level. Addition of $\mathrm{Ca}, \mathrm{P}, \mathrm{Mg}$ to the ration is recommended to equilibrate theire level changes during absorption from the intestine or by their exhaustion from bones due to their decrease in plasma of fluorosed birds. Fluorine exerted its toxic effect not only on bone and teeth but also on the elemental status and hormones such as thyroxin and triiodothyronine which needs more investigations on the human beings exposed to industrial fluorosis either in aluminum or superphosphate industry.

\section{REFERENCES}

Abd El-Nasser,M.;Seddek,A.sh.;Ahlam,A.and El-Saedy,I.(1995):

Immunotoxic effects of sodium fluoride in chickens. Assiut Vet.

Med.J.Volume 34 No.67:110-129. 
Ahlam, A.; Seddek, A.Sh.and Ibrahim, Th.A. (1994): Picture of endemic fluorosis in hens. Vet. Med. J., Giza, Vol. 42, No.1.295-304.

Balfield, A. and Goldberge, D.M. (1971): Colorimetric method for the determination of alkaline phosphatase activity. Enzyme 12: 561.

Becker, D.E.; Griffith, J.M.; Hobbs, C.S. and Maclntier, W.H. (1950): The alleviation of fluorine toxicosis by means of certain aluminum compounds. J. Animal Science, 9: 647-649.

Bett, I.M. and Fraser, C.P. (1959): Determination of calcium in serum Clin. Chem. Acta. 4: 364.

Brudevold, F.; Morino, E. and Bakhos, Y. (!972): Fluoride complexes in drinking water. Arch. Oral Biol. 17: 1155-1163.

Coles, E.H. (1988): Veterinary clinical pathology,4th ed.W.S.Sounder company, Philadilphia, London.

Deleers, M. (1985): Cationic atmosphere and cation competition binding at negatively charged membrane. Pathologicaimplications for aluminum. Res. Commun. Chem. Pathol. Pharmacol. 49 (2): 277294.

Douset, J.C.; Riofel, G.; Feliste, R.; Levy, P. and Bourbon, P. (1984): Effect of inhailed hydrogen fluoride on lipid metabolism in Guinea pigs. Fundam. App. Toxicol. 4: 618-623.

Fletcher, M.J.(1968): Clin.chem.Acta,22,393.cited from biomerieux triglyceride kit.

Fry, B.W. and Taves, D.R. (1970): Serum fluoride analysis with the fluoride electrodes. J. Lab. \& Clinic. Med., 75: 1020-1025.

Greenwood, D.A.; Shupe, J.L.; St, G.E.; Hariss, L.E.; Nielsen, H.M. and Ulsen, L.E. (1964): Flurosis icat. Agri. Expt.sta.utah state Uni.spechial report.

Grinder,E.and Heth,D.(1971): Determination of magnesium in serum and urine.clin.chem.17,662.

Hasamien, A.A., Ahlam, A., Abd El-Nasser,M., Seddek, A.Sh. and Ibrahim, Th.A. (1995): Effect of Fluoride toxicity on broiler chicken chromosomes. Vet. Med. J., Gizza, Vol. 43, No. 4, 421-427.

Hatfield, J.D.; Shewsburg, C.L. and Doyle, L.P. (1942): The effect of fluorine in rockphosphate in the nutrition of fattening lambs. J. Anim. Science, 1: 131-136.

Kalton, G. (1967): Introduction to statistical idess from socil scientist, $2 \underline{\text { nd }}$ Acad, Press, London.

Lotz, M.; Zisman, E. and Bartter, F.C. (1968): Evidence for a phosphorus depletion syndrome in man. N. Engl. J. Med., 274: 409-415. 
Majumdar, B.N. and Ray, S.N. (1946): Fluoride intoxication of cattle in India. III Effect of fluorosis on the composition of blood. Indian J. Vet Sci., 16: 113-121.

Morin, P.P.; Caroff, J.; Savina, A.; Thomas, J.; Labelles, M. and Morin, J.P. (1975): Determination of iodide in urine by ion selective electrode. Ann. Bil. Clin., 33: 89.

Morinl, L. and Prox, J. (1973): New and ragid procedure for serum phosphorus using of pheyiendiamine as reductant clin. Chem. Acta, 46: 113-117.

Potmann, M. (1979): Wirkungen von Kalziumfluoridreichen Ziegeleistauben auf Rinder (Ergebnisse Eines Einjahrigen Futtersversuches). $\mathrm{Ph}$.

D. Thesis , Giessen, Germany.

Rosenberger, G.; Dirksen, G.; Grunder, H.D.; Grunert, E.; Krause, D. and Stober, M. (1979): Clinical examination of cattle. Verlag. Paul Pary. Berlin and Hamburg.

Schmidt, H.J.; Newell, G.W. and Rand, W.E. (1954): The controlled feeding of fluorine, as NaF to dairy cattle, Am. J. Vet. Res. 15: 232-239.

Seddek, A.Sh.(1988): Clinico-toxicological study of environmental pollution of sulphur and fluorine in goat.Ph.D.Thesis,Faculty of Vet.Medicine,.Assiut University.

Shupe, J. L.; Miner, M.L.; Greenwood , D.A. Haris, L.E. and Stoddard, G.E. (1963): The effect of fluorine in dairy cattle.11-clinical and pathologic effects.Am.J.Vet.Res.,24(102):964-979.

Slagvold, L. (1934): Fluorforgiftning (german and english summary) O. Norsk Veterinaer- Tidsskr. 46-52.

Spencer, $H$. and Lender, M. (1979): Advers effects of aluminum containing antiacids on mineral metabolism. Gastroenterology, 76: 603-606.

Spencer, H.; Kramer, L. and Osis, D. (1980): Magnesium and alumon fluoride metabolism in man. In: Annual New York Academy of Sciences. Micro Nutient interactions: Vit. Min. and hazardouelements ed: O.A. Levander and Iorraine Chang. pp. 181-194.

Zoasoo, A. (1975): T4 thyrotoxicosis with normal or low serum T3 concentration. Aust. Nzj. Med., 5: 432-434. 


\begin{tabular}{|c|c|c|c|c|c|c|c|c|c|}
\hline 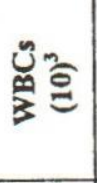 & 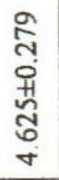 & 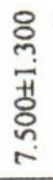 & 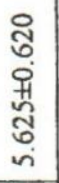 & 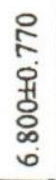 & 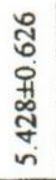 & \begin{tabular}{l}
$\approx$ \\
6 \\
0 \\
+ \\
\multirow{2}{*}{} \\
0
\end{tabular} & 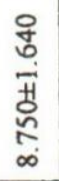 & 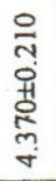 & 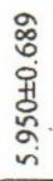 \\
\hline 岂。 & 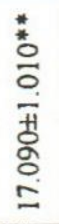 & 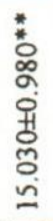 & $\begin{array}{l}= \\
\Xi \\
\text { से } \\
\dot{+} \\
\text { ते }\end{array}$ & 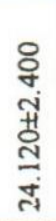 & 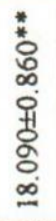 & 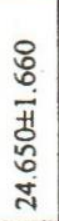 & 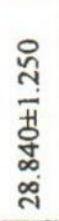 & 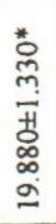 & 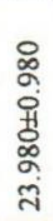 \\
\hline 总 & 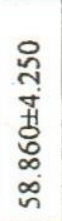 & 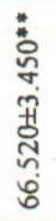 & 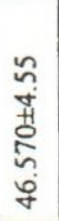 & 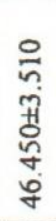 & 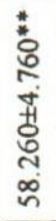 & 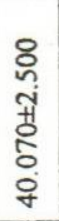 & 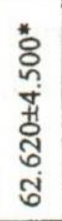 & $\begin{array}{l}8 \\
\delta \\
\text { ते } \\
\text { गे } \\
\infty \\
+ \\
\infty \\
+\end{array}$ & 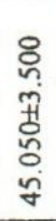 \\
\hline$\vec{z}$ & \begin{tabular}{l}
$\stackrel{*}{*}$ \\
\multirow{N}{*}{} \\
\multirow{H}{*}{} \\
$\stackrel{\infty}{0}$ \\
$\stackrel{\infty}{0}$ \\
$\stackrel{0}{n}$
\end{tabular} & 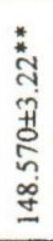 & 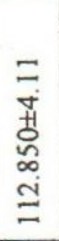 & 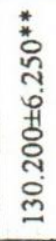 & 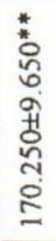 & 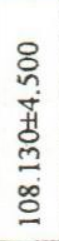 & 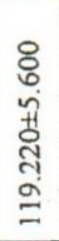 & 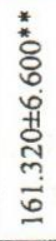 & 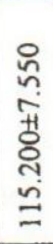 \\
\hline 己े & 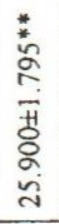 & 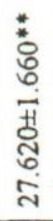 & 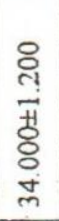 & 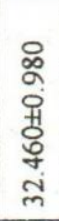 & 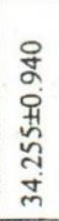 & $\begin{array}{l}\text { ¿ } \\
8 \\
0 \\
01 \\
0 \\
\infty \\
\infty \\
\text { m }\end{array}$ & 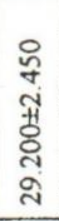 & 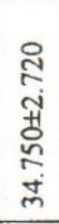 & 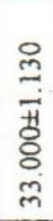 \\
\hline 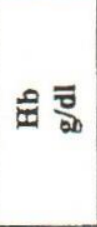 & $\begin{array}{l}\text { *0 } \\
0 \\
0 \\
0 \\
0 \\
01 \\
\text { + } \\
\Xi \\
=\end{array}$ & 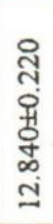 & 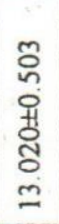 & 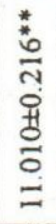 & 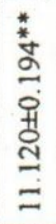 & 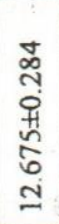 & 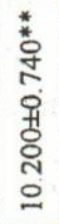 & 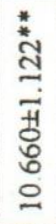 & 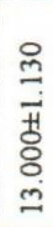 \\
\hline 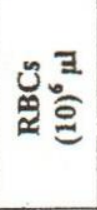 & $\begin{array}{l}\text { * } \\
\text { 음 } \\
\text { స̃ } \\
\text { 꽁 } \\
\stackrel{0}{\circ}\end{array}$ & 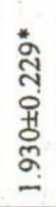 & 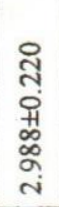 & 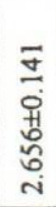 & 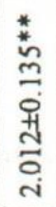 & 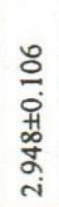 & 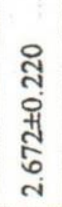 & 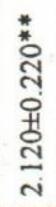 & 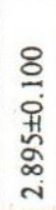 \\
\hline 产 & 8 & 8 & 을 & 8 & 8 & 홈 & ᄋ & ర్ & 홍 \\
\hline 总吾 & & $\sim$ & & & $N$ & & & m & \\
\hline
\end{tabular}




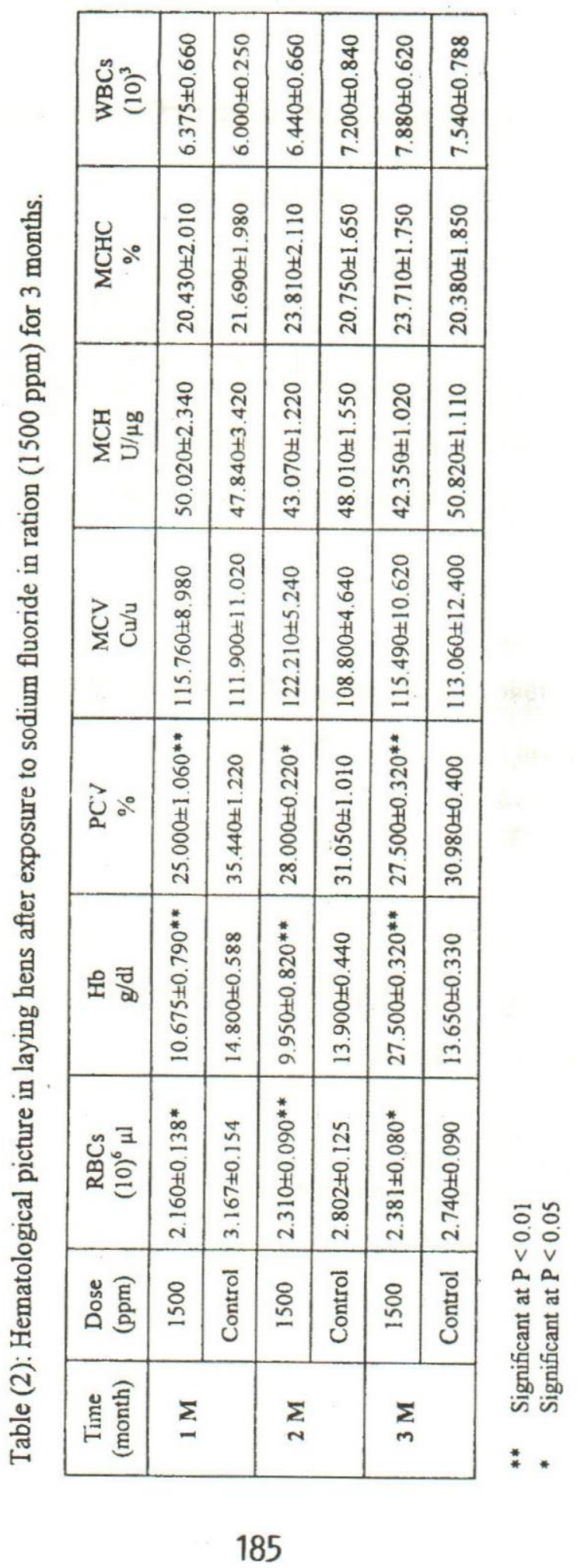


Table (3): Mean of egg weight / week in fluoretic and treated hens.

\begin{tabular}{|c|c|c|c|c|c|}
\hline \multirow{2}{*}{$\begin{array}{c}\text { Time } \\
\text { in } \\
\text { week }\end{array}$} & \multirow{2}{*}{ Control } & \multicolumn{2}{|c|}{1000 ppm } & \multicolumn{2}{c|}{1500 ppm } \\
\cline { 3 - 6 } & & F. & F. + AL & F. & F. + AL \\
\hline 1 & $37.060 \pm 0.085$ & $33.545 \pm 0.230^{* *}$ & $35.330 \pm 0.33^{*} \mathrm{D}$ & $33.666 \pm 0.650^{* *}$ & $37.500 \pm 0.960 \mathrm{D}$ \\
\hline 2 & $38.118 \pm 0.554$ & $32.428 \pm 0.286^{* *}$ & $35.230 \pm 0.110^{* *} \mathrm{D}$ & $37.800 \pm 0.186$ & $40.200 \pm 0.677$ \\
\hline 3 & $39.684 \pm 0.071$ & $33.875 \pm 0.208^{* *}$ & $37.180 \pm 0.660^{*} \mathrm{D}$ & $35.214 \pm 0.267^{* *}$ & $38.250 \pm 0.780 \mathrm{D}$ \\
\hline 4 & $38.810 \pm 0.026$ & $32.600 \pm 0.630^{* *}$ & $37.526 \pm 0.687 \mathrm{D}$ & $38.250 \pm 0.280$ & $38.312 \pm 0.117$ \\
\hline
\end{tabular}

Table (4): Mean of week egg production / hen in fluoretic and treated hens.

\begin{tabular}{|c|c|c|c|c|c|}
\hline \multirow{2}{*}{$\begin{array}{c}\text { Time } \\
\text { in } \\
\text { Week }\end{array}$} & Control & \multicolumn{2}{|c|}{1000 ppm } & \multicolumn{2}{c|}{1500 ppm } \\
\cline { 3 - 6 } & & F. & F. + AL & F. & F. + AL \\
\hline 1 & $2.428 \pm 0.182$ & $1.857 \pm 0.311$ & $1.200 \pm 0.163^{* *}$ & $0.600 \pm 0.157^{* *}$ & $0.400 \pm 0.097^{* *}$ \\
\hline 2 & $2.225 \pm 0.331$ & $1.125 \pm 0.195^{*}$ & $1.950 \pm 0.137 \mathrm{D}$ & $1.400 \pm 0.185^{*}$ & $1.050 \pm 0.086^{* *}$ \\
\hline 3 & $2.500 \pm 0.346$ & $1.125 \pm 0.066^{* *}$ & $2.550 \pm 0.210 \mathrm{D}$ & $1.400 \pm 0.173^{* *}$ & $1.400 \pm 0.141^{* *}$ \\
\hline 4 & $4.000 \pm 0.21$ & $0.800 \pm 0.133^{* *}$ & $2.966 \pm 0.298^{*} \mathrm{D}$ & $1.400 \pm 0.133^{* *}$ & $2.975 \pm 0.15^{* * \mathrm{D}}$ \\
\hline
\end{tabular}

** Significant at $\mathrm{P}<0.01$

* Significant at $P<0.05$

D Significant in comparison with treated hen at $P<0.01$ 


\begin{tabular}{|c|c|c|c|c|c|c|c|c|c|c|}
\hline & & 至 & $\underset{m}{n} \frac{0}{0}$ & 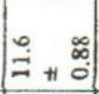 & 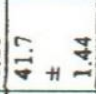 & 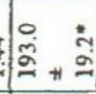 & Fे & 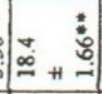 & $\sum_{\substack{0 \\
m+1}}^{\infty}$ & \\
\hline & ตั๊ & 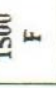 & $\underset{\sim}{\sim} \stackrel{\vdots}{\vdots}$ & $\stackrel{\infty}{=}$ - & 水 & 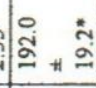 & 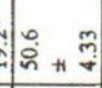 & $\stackrel{\text { aे }}{2}$ & ڤ్ & \\
\hline & & 吉 & 总 & 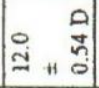 & $\stackrel{n}{n}$ & 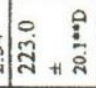 & (r) & 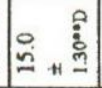 & i̊ & \\
\hline & ప & & ※. & 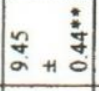 & 离 +1 ठ & 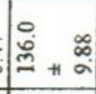 & $: \mid \vec{m}$ & 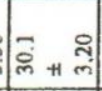 & 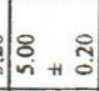 & \\
\hline & & ن & $\frac{a}{m+1}$ & $\overrightarrow{\tilde{m}}+\overrightarrow{0}$ & 足 & 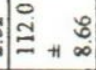 & $\vec{F}+\frac{9}{9}$ & $m_{n}^{\infty}$ & $\prod_{0}^{\infty}$ & \\
\hline & & 点点 & 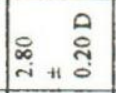 & 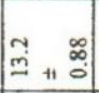 & $\frac{n}{m+1}=$ & 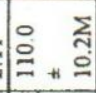 & 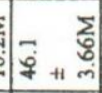 & $\mid$\begin{tabular}{ll}
0 & \\
\hdashline
\end{tabular} & $\overbrace{0}^{\infty}+1$ & \\
\hline & 4 & L & $\underset{\dot{0}}{\dot{0}}+\frac{\vdots}{0}$ & 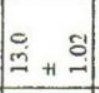 & 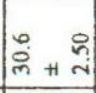 & 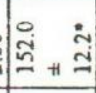 & 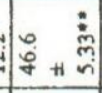 & 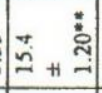 & 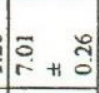 & 5 \\
\hline & & ti & $\underset{\substack{0 \\
ن+1}}{\vdots}$ & $\stackrel{0}{\stackrel{0}{m}}$ & 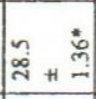 & 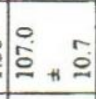 & 字 & 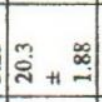 & {$\left[\begin{array}{l}\infty \\
\infty \\
n\end{array}\right.$} & 买告 \\
\hline & & m. & 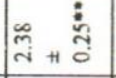 & $\stackrel{\infty}{\Xi}+\infty$ & $\overrightarrow{\bar{a}}+\vec{i}$ & {$\left[\begin{array}{ll}\infty \\
\mathbb{\Xi}\end{array}\right.$} & $\hat{m}$ & $\frac{r}{n}+\frac{0}{n}$ & 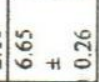 & 包 \\
\hline & & c & 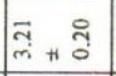 & $\begin{array}{ll}0 & 8 \\
& \end{array}$ & 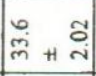 & $\cong$ & $\underset{j}{j}+\frac{j}{j}$ & $\stackrel{n}{\sim} \underset{-}{n}$ & $\stackrel{2}{2}$ + & \\
\hline & & & $\frac{a}{i}+\frac{0}{0}$ & 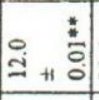 & 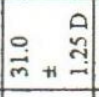 & 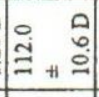 & 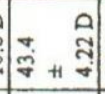 & 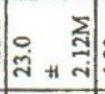 & & $\varangle$ \\
\hline & tै & & 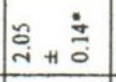 & 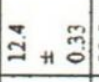 & 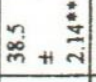 & $\mid$\begin{tabular}{cc}
0 & $\vdots$ \\
\hdashline \\
\hdashline
\end{tabular} & $\mid \begin{array}{ll}0 \\
0 \\
0\end{array}$ & 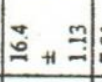 & 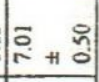 & \\
\hline & & $\begin{array}{l}4 \\
\pm\end{array}$ & $\frac{1}{4}+\frac{0}{0}$ & $\stackrel{0}{0}$ & 알 & 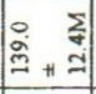 & 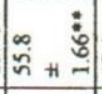 & {$\left[\begin{array}{ll}\infty & n \\
\vdots & 0\end{array}\right]$} & 궁 + & 2 \\
\hline & & set & 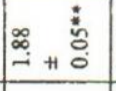 & $\bar{\Xi}$ \# & $\tilde{m}+$ & 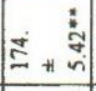 & $\tilde{n}+\begin{array}{ll}n \\
n\end{array}$ & {$\left[\begin{array}{ll}0 & 9 \\
\infty & 0\end{array}\right]$} & {$\left[\begin{array}{l}n \\
n \\
n\end{array}\right.$} & 劳写 \\
\hline & & & $\frac{\pi}{2}=$ & 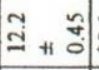 & $\overrightarrow{2}+\frac{n}{0}$ & $\varrho_{+}$is & 离 & 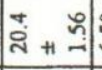 & 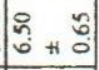 & ? \\
\hline & & 要 & 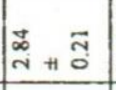 & 둘 & సุ. & 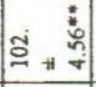 & 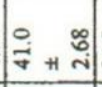 & 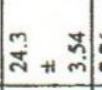 & $\stackrel{2}{2}$ & $*$ \\
\hline & & L & $\stackrel{a}{a}+\frac{7}{0}$ & 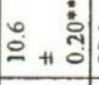 & 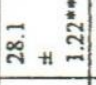 & ¿. & 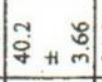 & $\begin{array}{ll}\infty \\
\dot{\sim} \\
\dot{n}\end{array}$ & 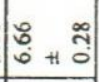 & \\
\hline & & 起 & 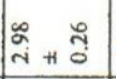 & $\exists$. & 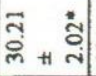 & $\begin{array}{cc}\vdots \\
0 \\
0\end{array}$ & 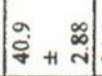 & $\left.\mid \begin{array}{ll}0 \\
\dot{a}\end{array}\right]$ & $\mid \begin{array}{cc}7 \\
6 \\
0\end{array}$ & \\
\hline & & 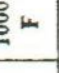 & $\stackrel{\substack{\infty \\
\infty}}{\substack{0 \\
\sim}}$ & ב̇ & 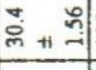 & ก & 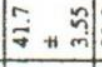 & $\underset{\sim}{2}+1$ & $\stackrel{n}{\circ}+\stackrel{5}{\circ}$ & 画 \\
\hline & & 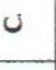 & $\stackrel{0}{0}+\frac{7}{m}$ & 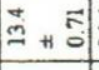 & $\vec{D}_{n+1}=$ & ปิ & $\begin{array}{ll}7 \\
7\end{array}$ & (ิ) & : & ठั 嵒 \\
\hline & & 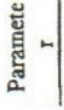 & 宅气 & 号 $\overline{0}$ & 己己ఃㅇ & 己 & \begin{tabular}{ll}
\multicolumn{1}{c}{} & $\infty$ \\
0 & 1 \\
$\sum$ & 0
\end{tabular} & 预 & 孚气 & 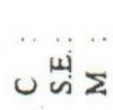 \\
\hline
\end{tabular}




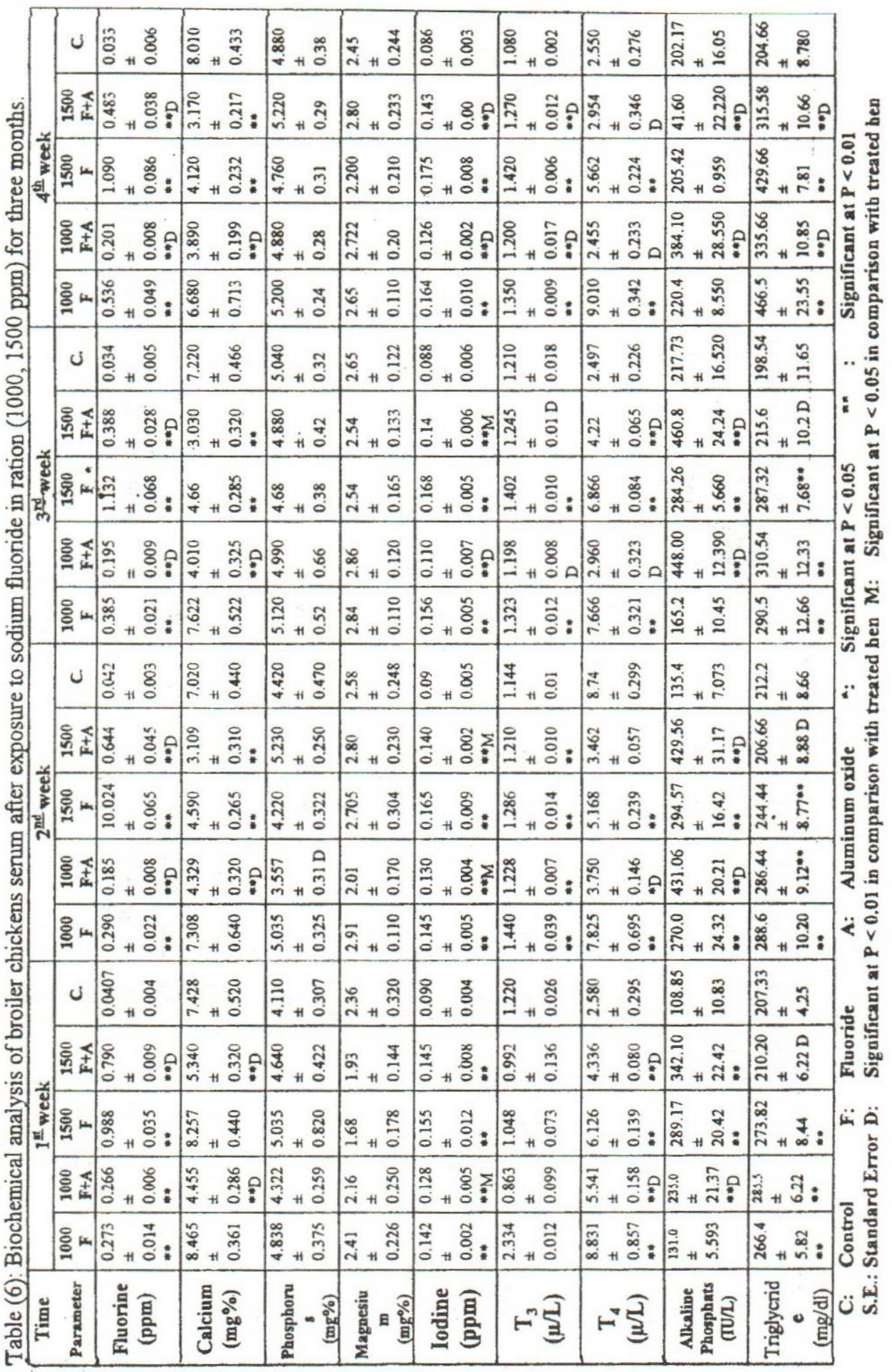




\begin{tabular}{|c|c|c|c|c|c|c|c|c|c|c|}
\hline \multirow{3}{*}{ 总 } & ద్ & 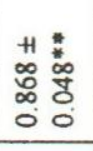 & 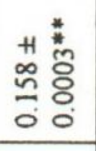 & 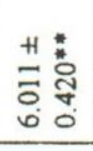 & 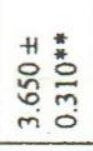 & $\begin{array}{ll}H & \\
0 & 8 \\
\infty & 8 \\
\infty & m \\
m & 0 \\
\end{array}$ & 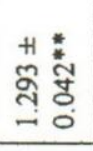 & 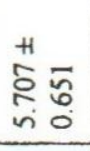 & 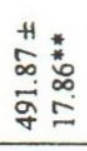 & 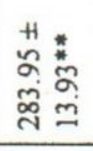 \\
\hline & ळ & $\begin{array}{ll}H & * \\
\infty & 0 \\
0 & 0 \\
& 0 \\
0 & 0\end{array}$ & 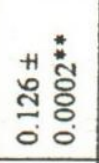 & 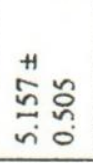 & 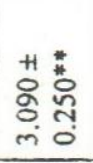 & $\begin{array}{l}H \\
\infty \\
\infty \\
+\end{array}$ & 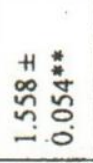 & 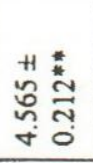 & $\begin{array}{l}+1 \\
8 \\
8 \\
\end{array}$ & 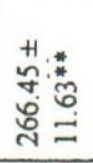 \\
\hline & $u$ & $\begin{array}{l}+1 \\
\infty \\
\infty \\
0 \\
0 \\
0\end{array}$ & 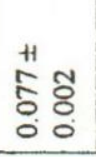 & $\begin{array}{l}+1 \\
0 \\
\text { N } \\
\infty \\
\infty \\
\infty \\
\infty\end{array}$ & 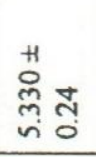 & $\begin{array}{l}\text { H } \\
\text { ơ } \\
\text { m } \\
\text { m o }\end{array}$ & $\begin{array}{l}+1 \\
\frac{\pi}{2} \\
0 \\
0\end{array}$ & $\begin{array}{l}+1 \\
\text { I } 0 \\
= \\
\text { Ni } 0 \\
\end{array}$ & $\begin{array}{l}+1 \\
\text { ñ } \\
\text { กู } \\
\text { กิ }\end{array}$ & 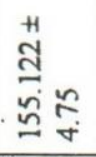 \\
\hline \multirow{3}{*}{ 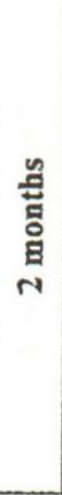 } & ఠ్ & 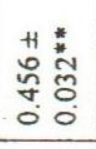 & 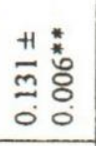 & 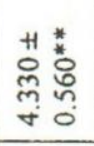 & 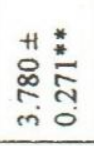 & 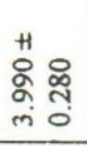 & 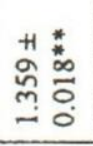 & 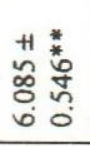 & 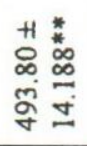 & 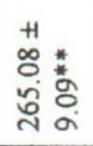 \\
\hline & छ & 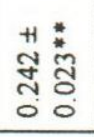 & 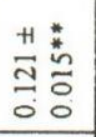 & 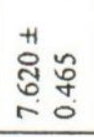 & 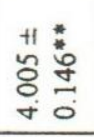 & $\begin{array}{l}+1 \\
0 \\
0\end{array}$ & 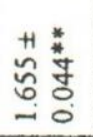 & 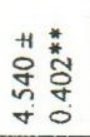 & 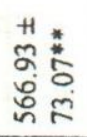 & 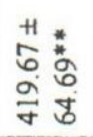 \\
\hline & $u$ & $\begin{array}{l}+1 \\
\infty \\
\text { đo } \\
0 \\
0\end{array}$ & $\begin{array}{l}+1 \\
\text { 용 } \\
0 \\
0\end{array}$ & 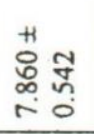 & 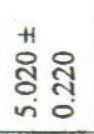 & 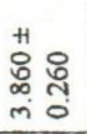 & 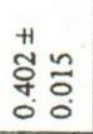 & 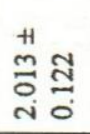 & 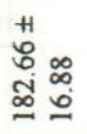 & 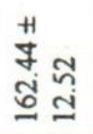 \\
\hline \multirow{3}{*}{ 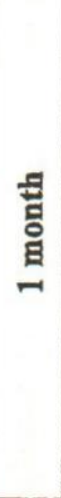 } & ڤ్ & 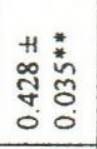 & 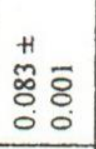 & 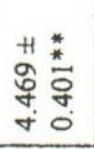 & 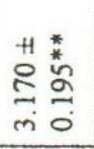 & 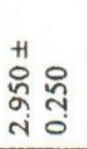 & 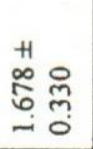 & 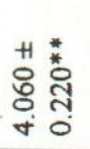 & 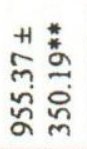 & 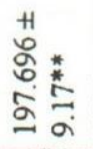 \\
\hline & 응 & 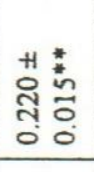 & 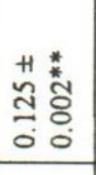 & $\begin{array}{l}+H \\
\infty \\
\sim \\
\infty \\
\infty\end{array}$ & $\begin{array}{l}+1 \\
\stackrel{8}{\circ} \frac{9}{1} \\
\text { ñ }\end{array}$ & 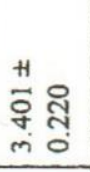 & 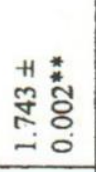 & 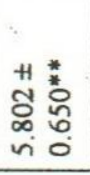 & 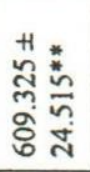 & 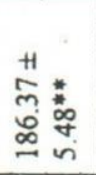 \\
\hline & $u$ & 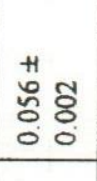 & $\begin{array}{ll}+1 & \text { m } \\
\text { ஸे } & \delta \\
0 & 0 \\
0 & 0\end{array}$ & 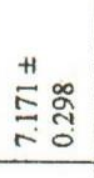 & 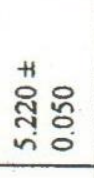 & $\begin{array}{l}+1 \\
\text { No } \\
\text { y } \\
\text { m }\end{array}$ & 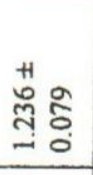 & 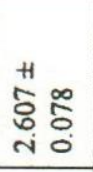 & 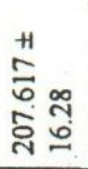 & $\begin{array}{l}\stackrel{H}{\cong} \\
\stackrel{\sim}{n} \frac{0}{\infty}\end{array}$ \\
\hline 茴 & 壱 & 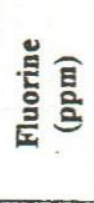 & 总合 & 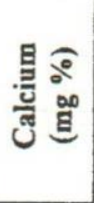 & 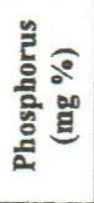 & 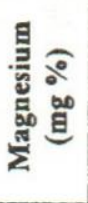 & - & $\Leftrightarrow$ 可 & 营 & 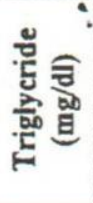 \\
\hline
\end{tabular}




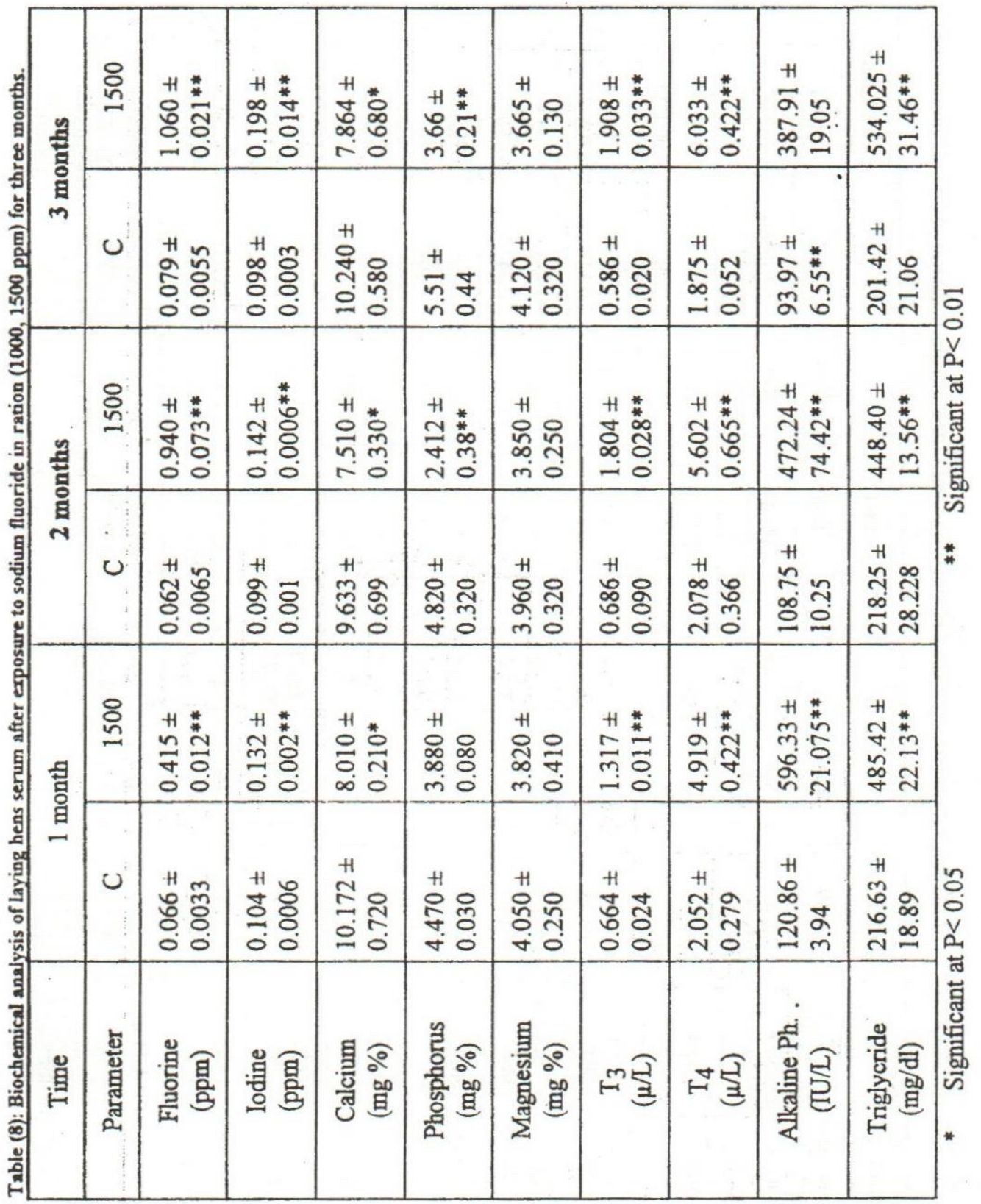




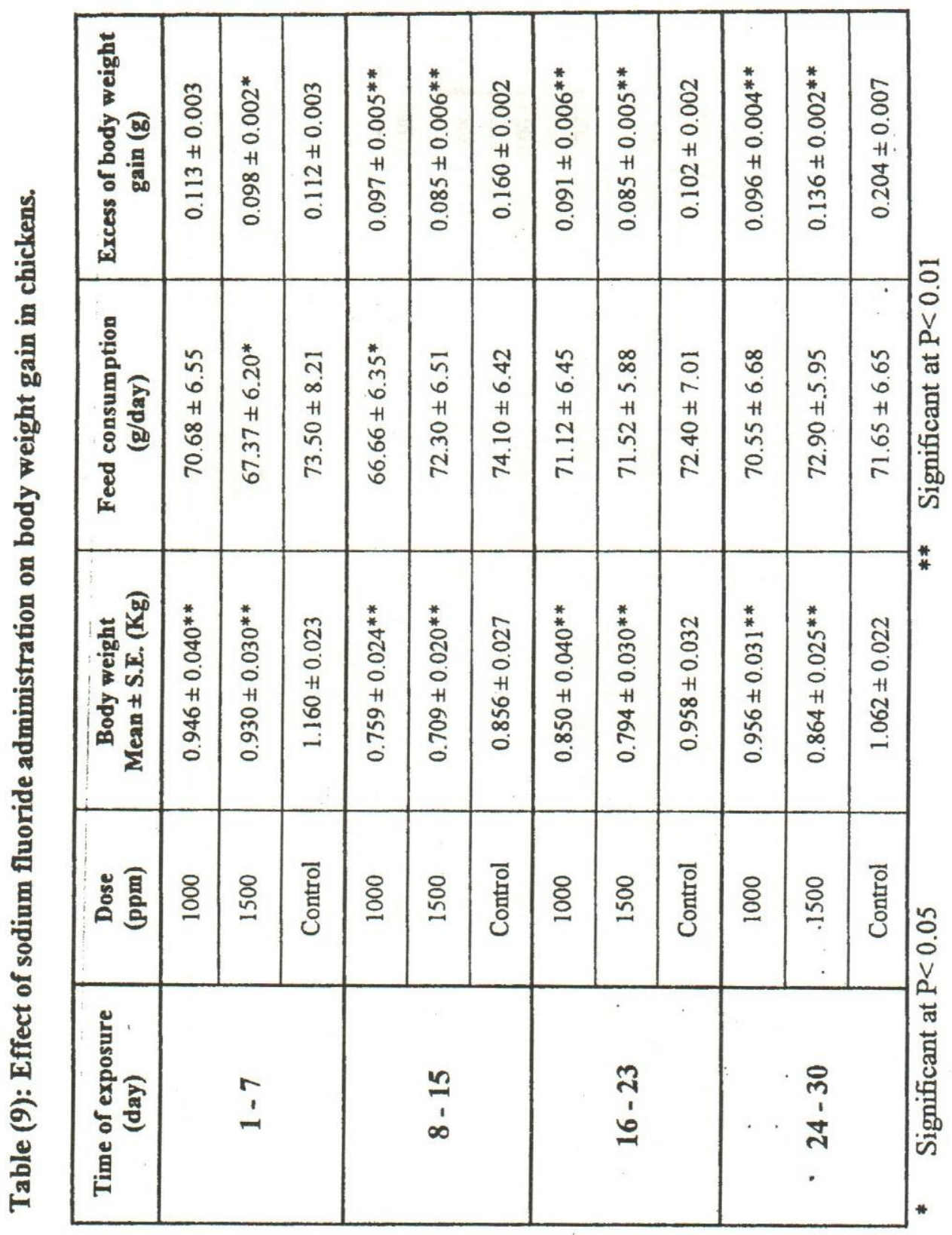




\begin{tabular}{|c|c|c|c|c|}
\hline \multirow{5}{*}{ 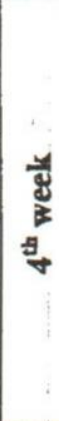 } & 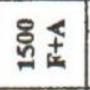 & $\stackrel{n}{=} \overrightarrow{0}$ & 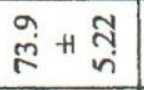 & $\stackrel{f}{\exists}$ \\
\hline & 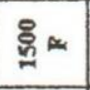 & $\tilde{\delta}+\tilde{-}$ & 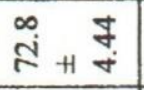 & ֶ̃ \\
\hline & 安走 & $\stackrel{\widetilde{m}}{-\widetilde{\delta}}$ & 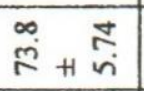 & $\stackrel{n}{+}+\stackrel{n}{+}$ \\
\hline & छ్రీజ & $\stackrel{\infty}{\infty}+10$ & $\stackrel{9}{\pi}+\underset{n}{n}$ & 官+ \\
\hline & ن & $\stackrel{\Xi}{\leftrightarrows}$ & 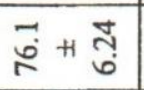 & 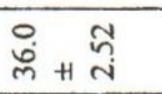 \\
\hline \multirow{5}{*}{ 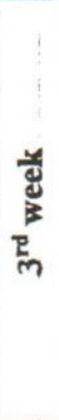 } & 量全 & $\stackrel{g}{\circ} \stackrel{0}{0}$ & $\stackrel{m}{i}+1 \vec{\exists}$ & $\stackrel{n}{m}+\vec{i}$ \\
\hline & 商 & $\overrightarrow{0}+\underset{i}{0}$ & $\overrightarrow{\vec{N}}_{H} \stackrel{\stackrel{\circ}{+}}{+}$ & 宫 \\
\hline & 总去 & 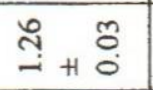 & $\vec{m}+\stackrel{\vec{r}}{+}$ & 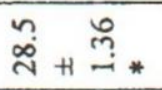 \\
\hline & 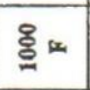 & 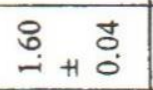 & i $+\pi$ & $\overrightarrow{\vec{N}}+\vec{N}$ \\
\hline & ن ن & $\stackrel{g}{g} \stackrel{0}{\circ}$ & 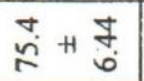 & లేm \\
\hline \multirow{5}{*}{ 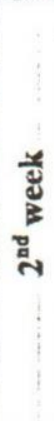 } & 影去 & $\stackrel{\infty}{\circ}+\infty \stackrel{0}{0}$ & $\stackrel{n}{2}+\frac{\overrightarrow{6}}{6}$ & 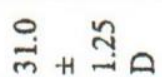 \\
\hline & 总地 & $\stackrel{\tilde{S}}{\stackrel{m}{\circ}}$ & $\stackrel{9}{\vec{R}} \stackrel{\stackrel{0}{+}}{+}$ & $\underset{m}{\infty} \underset{m}{\stackrel{\Xi}{N}}$ \\
\hline & 䲩吉 & 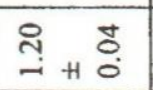 & 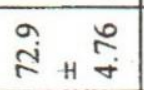 & $\underset{\dot{m}}{\stackrel{+}{+}}$ \\
\hline & ప్రీ & $\stackrel{\Xi}{\leftrightarrows}$ & $\stackrel{0}{i}+\stackrel{n}{i}$ & 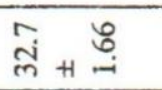 \\
\hline & ن & 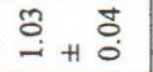 & 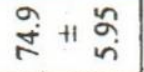 & $\overrightarrow{\hat{i}}+\stackrel{n}{0}$ \\
\hline \multirow{5}{*}{ E } & 总委 & 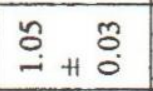 & $\stackrel{n}{n}+\bar{z}$ & $\stackrel{\text { Tे }}{n}+\frac{n}{n}$ \\
\hline & 鹿 & $\underset{-}{\tilde{D}}+\stackrel{\tilde{\sigma}}{0}$ & 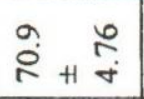 & $\vec{\sim} \underset{\sim}{\infty}$ Iี * \\
\hline & 部䘮 & $\stackrel{\infty}{\stackrel{\infty}{-}} \stackrel{\overrightarrow{0}}{0}$ & $\stackrel{0}{i}+\stackrel{\ddots}{b}$ & ণ̊ \\
\hline & \&్రీ & $\cong \underset{H}{\sigma}$ & $\stackrel{a}{r}+\stackrel{2}{n}$ & 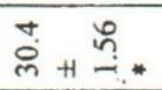 \\
\hline & ن & 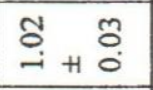 & $\stackrel{n}{n}+1=$ & $\vec{\phi}_{+1}=$ \\
\hline 目 & 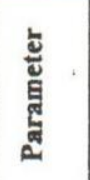 & 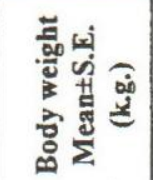 & 总 & 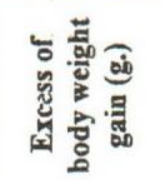 \\
\hline
\end{tabular}

